\title{
TAIL BEHAVIOR OF NEGATIVELY ASSOCIATED HEAVY-TAILED SUMS
}

\author{
JAAP GELUK, ${ }^{*}$ The Petroleum Institute, Abu Dhabi \\ KAI W. NG, ${ }^{* *}$ University of Hong Kong
}

\begin{abstract}
Consider a sequence $\left\{X_{k}, k \geq 1\right\}$ of random variables on $(-\infty, \infty)$. Results on the asymptotic tail probabilities of the quantities $S_{n}=\sum_{k=0}^{n} X_{k}, X_{(n)}=\max _{0 \leq k \leq n} X_{k}$, and $S_{(n)}=\max _{0 \leq k \leq n} S_{k}$, with $X_{0}=0$ and $n \geq 1$, are well known in the case where the random variables are independent with a heavy-tailed (subexponential) distribution. In this paper we investigate the validity of these results under more general assumptions. We consider extensions under the assumptions of having long-tailed distributions (the class $\mathrm{L}$ ) and having the class $\mathrm{D} \cap \mathrm{L}$, where $\mathrm{D}$ is the class of distribution functions with dominatedly varying tails. Some results are also given in the case where $X_{k}, k \geq 1$, are not necessarily identically distributed and/or independent.
\end{abstract}

Keywords: Asymptotics; subexponentiality; partial sum; tail probability; negative association

2000 Mathematics Subject Classification: Primary 60G50

Secondary $62 \mathrm{E} 20$

\section{Introduction}

The asymptotic tail behavior of sums of heavy-tailed random variables has been studied by many authors. Recent applications cover areas from importance sampling [3], where tail probabilities of sums are estimated, and queueing systems [5], [23], to nonparametric regression [16].

Although there does not seem to be general agreement on the terminology, a common feature of heavy-tailed random variables $X$ (or the corresponding distribution function $F$ ) which satisfy $\bar{F}(x)=\mathrm{P}\{X>x\}>0$, for any $x \in(-\infty, \infty)$, is the property $\operatorname{E} \exp \{\gamma X\}=\infty$, for any $\gamma>0$.

Early asymptotic results for the tail probability of the convolution of random variables with a regularly varying tail distribution (denoted by $F \in \mathrm{RV}$ ), i.e. for which there exists an $\alpha>0$ such that

$$
\bar{F}(t x) \sim t^{-\alpha} \bar{F}(x), \quad t>0,
$$

are due to Feller [9]. Here and henceforth, all limiting relationships are for $x \rightarrow \infty$ unless stated otherwise; by using the symbol ' $\sim$ ' we mean that the ratio of the two sides tends to 1 .

Received 19 September 2005; revision received 11 November 2005.

* Postal address: The Petroleum Institute, PO Box 2533, Abu Dhabi, United Arab Emirates.

Email address: jgeluk@pi.ac.ae

** Postal address: Department of Statistics and Actuarial Science, University of Hong Kong, Pokfulam Road, Hong Kong. Email address: kaing@hku.hk 
The study of the asymptotic behavior of tail sums was (see [13] and references therein) subsequently extended to the class of subexponential distributions, S. By definition, a distribution function $F$ on $[0, \infty)$ is defined to be subexponential (denoted by $F \in S$ ) if

$$
\frac{\bar{F}^{* 2}(x)}{\bar{F}(x)} \rightarrow 2
$$

where $\bar{F}^{* 2}(x)=\mathrm{P}\left\{X_{1}+X_{2}>x\right\}$ is the tail probability of the convolution of two independent and identically distributed (i.i.d.) random variables with distribution function $F$. More generally, a distribution function $F$ on $(-\infty, \infty)$ is said to be subexponential if $F^{+}(x)=$ $F(x) \mathbf{1}_{\{0 \leq x<\infty\}}$ is subexponential, where $\mathbf{1}_{\{\cdot\}}$ is the indicator function.

In this case it is possible to prove basic results, for example the fact that (1.1) implies that

$$
\frac{\bar{F}^{* n}(x)}{\bar{F}(x)} \rightarrow n, \quad \text { for } n \geq 2,
$$

for random variables on the real line; see, e.g. [10], [15], and [22]. For background information on subexponentiality and its applications, the reader is referred to [2], [4], [8], [18].

An important reason why subexponential distributions play a role in the fields of applied probability and risk theory is that, for a sequence of i.i.d. random variables with common distribution $F \in \mathrm{S}$, from (1.2) it holds, for each $n \geq 2$, that

$$
\mathrm{P}\left\{\sum_{k=1}^{n} X_{k}>x\right\} \sim \mathrm{P}\left\{\max _{1 \leq k \leq n} X_{k}>x\right\}
$$

which makes it clear that the class $\mathrm{S}$ is useful in modeling large losses.

The class $\mathrm{S}$ is related to several other classes of functions. A well-known result is the inclusion $\mathrm{S} \subset \mathrm{L}$, where $\mathrm{L}$ is the class of long-tailed distribution functions $F$ satisfying $\bar{F}(x+a) \sim \bar{F}(x)$, for $a \in \mathbb{R}$. In this case, convergence is uniform on compact subsets of $\mathbb{R}$.

There is a connection with functions of dominated variation as well: the inclusion $\mathrm{D} \cap \mathrm{L} \subset \mathrm{S}$. We write $F \in \mathrm{D}$ if the tail function $\bar{F}$ is of dominated variation, i.e. if $\limsup _{x \rightarrow \infty} \bar{F}(a x) / \bar{F}(x)<\infty$, for $0<a<1$. Convolution tails for dominatedly varying distributions were studied by Tang and Yan [20].

We use the following notation. For a sequence $\left\{X_{k}, k \geq 1\right\}$ of random variables with distribution functions on $(-\infty, \infty)$, using the convention $X_{0}=0$, we write

$$
X_{(n)}=\max _{0 \leq k \leq n} X_{k}, \quad S_{n}=\sum_{k=0}^{n} X_{k}, \quad S_{(n)}=\max _{0 \leq k \leq n} S_{k} .
$$

In this paper, under the assumption that the random variables $X_{k}, k \geq 1$, have heavy-tailed distribution functions, we aim to formulate the asymptotic relations

$$
\mathrm{P}\left\{S_{(n)}>x\right\} \sim \mathrm{P}\left\{X_{(n)}>x\right\} \sim \mathrm{P}\left\{S_{n}>x\right\} \sim \sum_{k=1}^{n} \bar{F}_{k}(x) .
$$




\section{The case of independent random variables}

Using the Pollaczek-Spitzer identity, Sgibnev [19] studied the asymptotic behavior of the tail probability of $S_{(n)}$ in the case of i.i.d. summands. In particular, when $F \in \mathrm{S}$, he proved that $\mathrm{P}\left\{S_{(n)}>x\right\} \sim n \bar{F}(x)$. Using a quantile inequality for $S_{n}$ from [17], a simplified proof of this equivalence was given in [14]. See also [13, Theorem 3.1] for the i.i.d. case.

Since (1.2) holds for distribution functions $F \in \mathrm{S}$, we have

$$
\mathrm{P}\left\{S_{(n)}>x\right\} \sim \mathrm{P}\left\{S_{n}>x\right\} \sim n \bar{F}(x) .
$$

Below we give a new proof which avoids the use of complicated technical results and is valid for nonidentically distributed random variables as well.

Theorem 2.1. Suppose that $X_{k}, k=1, \ldots, n$, are independent random variables with distribution functions $F_{1}, \ldots, F_{n}$ on $(-\infty, \infty)$.

(i) If $F_{i} \in \mathrm{L}$, for $i=1, \ldots, n$, then $\mathrm{P}\left\{S_{(n)}>x\right\} \sim \mathrm{P}\left\{S_{n}>x\right\}$.

(ii) Suppose that the convolution $F_{i} * F_{j}$ is a member of $\mathrm{S}$, for all $1 \leq i, j \leq n$, and one of the following conditions holds:

(iia) there exists a $c$ such that $\mathrm{P}\left\{X_{i}>c\right\}=1$, for $1 \leq i \leq n$,

(iib) $F_{i} \in \mathrm{L}$ for $i=1, \ldots, n$.

Then the asymptotic relations in (1.3) hold.

For every real number $x$ we write $x^{+}=x \vee 0=\max \{x, 0\}$. To prove Theorem 2.1 we need the following lemma.

Lemma 2.1. The following statements hold.

(i) If $F_{1}, F_{2} \in \mathrm{L}$ then $F_{1} * F_{2} \in \mathrm{L}$.

(ii) If $X_{i} \geq 0$ almost surely for $i=1, \ldots, n$ and $F_{i} * F_{j} \in \mathrm{S}$ for all $1 \leq i, j \leq n$, then $F_{k} \in \mathrm{S}$ and $F_{1} * F_{2} \cdots * F_{k} \in \mathrm{S}$ for $1 \leq k \leq n$, and $\mathrm{P}\left\{S_{n}>x\right\} \sim \sum_{k=1}^{n} \bar{F}_{k}(x)$.

(iii) If $F_{1}, F_{2} \in \mathrm{L}$ then $\mathrm{P}\left\{X_{1}^{+}+X_{2}>x\right\} \sim \mathrm{P}\left\{X_{1}+X_{2}>x\right\}$.

Proof. Part (i) is from [6]; see also [14]. The convolution closure of S is from [7]. The other results of parts (ii) and (iii) are from [11] and [10], respectively.

Proof of Theorem 2.1. We use the notation $f(x) \lesssim g(x)$ to mean $\lim \sup f(x) / g(x) \leq 1$, and define the reverse relation in the natural way. We use induction to prove that

$$
\mathrm{P}\left\{S_{(n)}>x\right\} \sim \mathrm{P}\left\{S_{n}>x\right\} .
$$

Suppose that $F_{i} \in \mathrm{L}$, for $i=1, \ldots, n$, and that (2.1) holds for all values of subscript less than or equal to $n-1$. The inequality $\mathrm{P}\left\{S_{(n)}>x\right\} \gtrsim \mathrm{P}\left\{S_{n}>x\right\}$ is trivial, so we only need to find 
an upper estimate. Note that

$$
\begin{aligned}
\mathrm{P}\left\{S_{(n)} \geq x\right\} & =\sum_{k=1}^{n} \mathrm{P}\left\{S_{0} \leq x, \ldots, S_{k-1} \leq x, S_{k}>x\right\} \\
& \leq \sum_{k=1}^{n} \mathrm{P}\left\{S_{k-1} \leq x, X_{k}+S_{k-1}>x\right\} \\
& =\sum_{k=1}^{n}\left[\mathrm{P}\left\{S_{k}>x\right\}-\mathrm{P}\left\{S_{k-1}>x\right\}+\mathrm{P}\left\{S_{k-1}>x, S_{k} \leq x\right\}\right] \\
& =\mathrm{P}\left\{S_{n}>x\right\}+\sum_{k=1}^{n} \mathrm{P}\left\{S_{k-1}>x, S_{k} \leq x\right\} .
\end{aligned}
$$

Since the class L is closed under convolution by Lemma 2.1(i), we may apply Lemma 2.1(iii) to find that

$$
\mathrm{P}\left\{S_{k-1}>x, X_{k}+S_{k-1} \leq x\right\}=\mathrm{P}\left\{S_{k-1}+X_{k}^{+}>x\right\}-\mathrm{P}\left\{S_{k-1}+X_{k}>x\right\}=o\left(\mathrm{P}\left\{S_{k}>x\right\}\right) .
$$

Hence,

$$
\mathrm{P}\left\{S_{(n)} \geq x\right\} \leq \mathrm{P}\left\{S_{n}>x\right\}+o\left(\sum_{k=1}^{n} \mathrm{P}\left\{S_{k}>x\right\}\right) .
$$

Since $\mathrm{P}\left\{S_{(k)}>x\right\}$ is nondecreasing in $k$ for all $x$, using the induction hypothesis $\mathrm{P}\left\{S_{k}>x\right\} \sim$ $\mathrm{P}\left\{S_{(k)}>x\right\}$ for $k \leq n-1$ it follows that

$$
o\left(\sum_{k=1}^{n} \mathrm{P}\left\{S_{k}>x\right\}\right)=o\left(\mathrm{P}\left\{S_{n}>x\right\}\right)+o\left(\mathrm{P}\left\{S_{(n)}>x\right\}\right) .
$$

Combining this with (2.2) gives $\mathrm{P}\left\{S_{(n)} \geq x\right\} \lesssim \mathrm{P}\left\{S_{n} \geq x\right\}$, completing the proof of part (i).

In order to prove part (ii) under the assumption given by part (iia), note that (2.1) holds, since the assumption $F_{i} * F_{j} \in \mathrm{S}$, for all $1 \leq i, j \leq n$, implies that $F_{i} \in \mathrm{S} \subset \mathrm{L}$ (consider Lemma 2.1(ii) applied to $X_{i}-c$ ).

Next we prove part (ii) under the assumption given by part (iib). Note that application of Lemma 2.1(iii) gives $\mathrm{P}\left\{X_{1}+X_{2}>x\right\} \sim \mathrm{P}\left\{X_{1}^{+}+X_{2}^{+}>x\right\}$, which implies that the distribution function of $X_{1}^{+}+X_{2}^{+}$is subexponential (since the tail is asymptotic to $\mathrm{P}\left\{S_{2}>x\right\}$ ). Using part (iia) above, the right-hand side of (2.1) is asymptotic to

$$
\sum_{k=1}^{2} \mathrm{P}\left\{X_{k}^{+}>x\right\}=\sum_{k=1}^{2} \mathrm{P}\left\{X_{k}>x\right\} .
$$

The proof for $n>2$ follows by induction.

In view of Lemma 2.1(ii) and $\mathrm{P}\left\{X_{(n)}>x\right\}=1-\prod_{k=1}^{n}\left(1-\bar{F}_{k}(x)\right) \sim \sum_{k=1}^{n} \bar{F}_{k}(x)$, the proof is complete.

\section{The case of negatively associated random variables}

A finite family of random variables $\left\{X_{k}, 1 \leq k \leq n\right\}$ is said to be negatively associated (NA) if, for every pair of disjoint subsets $A_{1}$ and $A_{2}$ of $\{1,2, \ldots, n\}$,

$$
\operatorname{cov}\left\{f_{1}\left(X_{k_{1}}, k_{1} \in A_{1}\right), f_{2}\left(X_{k_{2}}, k_{2} \in A_{2}\right)\right\} \leq 0,
$$


whenever $f_{1}$ and $f_{2}$ are coordinatewise increasing such that the covariance exists. An infinite family is NA if each of its finite subfamilies is NA. This dependence structure was first introduced in [1] and [12].

Theorem 3.1. Suppose that the random variables $X_{k}, k=1, \ldots, n$, are NA with distribution functions

$$
F_{k} \in \mathrm{D} \cap \mathrm{L}, \quad k \geq 1,
$$

and there exists a constant $c>-\infty$ such that $\mathrm{P}\left\{X_{k}>c\right\}=1, k=1, \ldots, n$. Then (1.3) holds.

Wang and Tang [21] established the same result under the additional conditions that $X_{1}, \ldots, X_{n}$ are identically distributed and $\mathrm{E} X_{1}^{r}<\infty$, for some $r>1$.

A closer look at the proof of Theorem 3.1 shows that it is sufficient to assume the asymptotic version of the NA property given below, because the inequalities need to be satisfied for large values of $x$ only. A finite family of random variables $\left\{X_{k}, 1 \leq k \leq n\right\}$ is said to be asymptotically $N A$ if there exists a constant $c_{0}$ such that, for every pair of disjoint subsets $A_{1}$ and $A_{2}$ of $\{1,2, \ldots, n\}$,

$$
\operatorname{cov}\left\{f_{1}\left(X_{k_{1}}, k_{1} \in A_{1}\right), f_{2}\left(X_{k_{2}}, k_{2} \in A_{2}\right)\right\} \leq 0,
$$

for functions $f_{1}$ and $f_{2}$ which have support $\left(c_{0}, \infty\right)$ and are coordinatewise increasing such that the covariance exists.

Proof of Theorem 3.1. The proof consists of three parts.

(i) We start by proving that $\mathrm{P}\left\{S_{n}>x\right\} \sim \sum_{k=1}^{n} \bar{F}_{k}(x)$, which amounts to a conjunction of the two asymptotic relations

$$
\mathrm{P}\left\{S_{n}>x\right\} \gtrsim \sum_{k=1}^{n} \bar{F}_{k}(x) \text { and } \mathrm{P}\left\{S_{n}>x\right\} \lesssim \sum_{k=1}^{n} \bar{F}_{k}(x) .
$$

Choose functions $a_{k}(x) \uparrow \infty$ such that $\bar{F}_{k}\left(x \pm a_{k}(x)\right) \sim \bar{F}_{k}(x)$, for $k=1, \ldots, n$. It follows that, with $a(x)=\min _{1 \leq k \leq n} a_{k}(x)$,

$$
\bar{F}_{k}(x \pm a(x)) \sim \bar{F}_{k}(x), \quad k=1, \ldots, n .
$$

Note that

$$
\begin{aligned}
\mathrm{P}\left\{S_{n}>x\right\} \geq & \mathrm{P}\left\{S_{n}>x, X_{(n)}>x+a(x)\right\} \\
\geq & \sum_{k=1}^{n} \mathrm{P}\left\{S_{n}>x, X_{k}>x+a(x)\right\} \\
& -\sum_{1 \leq k<l \leq n} \mathrm{P}\left\{S_{n}>x, X_{k}>x+a(x), X_{l}>x+a(x)\right\} \\
= & : J_{1}-J_{2} .
\end{aligned}
$$

Since $\mathrm{P}\left\{X_{k}>c\right\}=1$, for $k=1, \ldots, n$, it holds for $x$ sufficiently large that

$$
J_{1} \geq \sum_{k=1}^{n} \mathrm{P}\left\{S_{n}-X_{k}>-a(x), X_{k}>x+a(x)\right\}=\sum_{k=1}^{n} \bar{F}_{k}(x+a(x)) .
$$


In view of (3.2), we have $J_{1} \gtrsim \sum_{k=1}^{n} \bar{F}_{k}(x)$. Using the NA property, it follows that

$$
J_{2} \leq \sum_{1 \leq k<l \leq n} \mathrm{P}\left\{X_{k}>x+a(x), X_{l}>x+a(x)\right\} \leq \sum_{1 \leq k<l \leq n} \bar{F}_{k}(x+a(x)) \bar{F}_{l}(x+a(x)) ;
$$

hence, $J_{2}=o\left(\sum_{k=1}^{n} \bar{F}_{k}(x)\right)$. This proves the first relation in (3.1).

To prove the second relation in (3.1) in terms of the function $a(x)$ in (3.2), we derive

$$
\begin{aligned}
\mathrm{P}\left\{S_{n}>x\right\} \leq & \mathrm{P}\left\{\bigcup_{k=1}^{n} X_{k}>x-a(x)\right\}+\mathrm{P}\left\{S_{n}>x, \bigcap_{k=1}^{n}\left[X_{k} \leq x-a(x)\right]\right\} \\
\leq & \sum_{k=1}^{n} \mathrm{P}\left\{X_{k}>x-a(x)\right\} \\
& +\mathrm{P}\left\{S_{n}>x, \bigcup_{k=1}^{n}\left[X_{k}>\frac{x}{n}\right], \bigcap_{k=1}^{n}\left[X_{k} \leq x-a(x)\right]\right\} .
\end{aligned}
$$

By the choice of the function $a$, the first term on the right-hand side is asymptotic to $\sum_{k=1}^{n} \bar{F}_{k}(x)$. Clearly, the second term is dominated by $\sum_{k=1}^{n} \mathrm{P}\left\{S_{n}-X_{k}>a(x), X_{k}>x / n\right\}$. Using the NA property, it follows that this expression is further dominated by

$$
\sum_{k=1}^{n} \mathrm{P}\left\{S_{n}-X_{k}>a(x)\right\} \mathrm{P}\left\{X_{k}>\frac{x}{n}\right\}=o\left(\sum_{k=1}^{n} \bar{F}_{k}(x)\right),
$$

the last equality being true since $F_{k} \in \mathrm{D}$, for $1 \leq k \leq n$. This proves the second relation in (3.1).

(ii) Next we prove that

$$
\mathrm{P}\left\{X_{(n)}>x\right\} \sim \sum_{k=1}^{n} \bar{F}_{k}(x) .
$$

The inequality $\mathrm{P}\left\{X_{(n)}>x\right\} \leq \sum_{k=1}^{n} \bar{F}_{k}(x)$, for all $x$, is trivial. To prove the reverse relation, by the NA property we have

$$
\mathrm{P}\left\{X_{(n)}>x\right\} \geq \sum_{k=1}^{n} \mathrm{P}\left\{X_{k}>x\right\}-\sum_{1 \leq k \neq l \leq n} \mathrm{P}\left\{X_{k}>x, X_{l}>x\right\} \sim \sum_{k=1}^{n} \bar{F}_{k}(x) .
$$

(iii) Finally we prove (2.1). Note that part (i) of this proof implies that

$$
\mathrm{P}\left\{S_{(n)}>x\right\} \leq \mathrm{P}\left\{\sum_{i=1}^{n} X_{i}^{+}>x\right\} \lesssim \sum_{i=1}^{n} \mathrm{P}\left\{X_{i}^{+}>x\right\}=\sum_{i=1}^{n} \bar{F}_{i}(x) .
$$

Since $\mathrm{P}\left\{S_{(n)}>x\right\} \geq \mathrm{P}\left\{S_{n}>x\right\}$, the proof is complete.

\section{Acknowledgements}

The authors would like to thank Dr Qihe Tang, who made helpful comments on an earlier draft of this paper. The final version of this paper was completed during a research visit of the first author to Hong Kong University. Grateful acknowledgement is made to the Department of Statistics and Actuarial Science there for hospitality and support. 


\section{References}

[1] Alam, K. and Saxena, K. M. L. (1981). Positive dependence in multivariate distributions. Commun. Statist. A 10, 1183-1196.

[2] Asmussen, S. (2000). Ruin Probabilities (Adv. Ser. Statist. Sci. Appl. Prob. 2). World Scientific, River Edge, NJ.

[3] Asmussen, S., Kroese, D. P. and Rubinstein, R. Y. (2005). Heavy tails, importance sampling and cross-entropy. Stoch. Models 21, 57-76.

[4] Bingham, N. H., Goldie, C. M. And Teugels, J. L. (1987). Regular Variation (Encyclopedia Math. Appl. 27). Cambridge University Press.

[5] Boxma, O. J., Foss, S. G., Lasgouttes, J.-M. and Núñez QueiJa, R. (2004). Waiting time asymptotics in the single server queue with service in random order. Queueing Systems 46, 35-73.

[6] Embrechts, P. And Goldie, C. M. (1980). On closure and factorization properties of subexponential and related distributions. J. Austral. Math. Soc. Ser. A 29, 243-256.

[7] Embrechts, P., Goldie, C. M. and Veraverbeke, N. (1979). Subexponentiality and infinite divisibility. Z. Wahrscheinlichkeitsth. 49, 335-347.

[8] Embrechts, P., Klüppelberg, C. and Mikosch, T. (1997). Modelling Extremal Events (Appl. Math. (New York) 33). Springer, Berlin.

[9] Feller, W. (1971). An Introduction to Probability Theory and Its Applications, Vol. 2, 2nd edn. John Wiley, New York.

[10] Geluk, J. (2004). Asymptotics in the symmetrization inequality. Statist. Prob. Lett. 69, 63-68.

[11] GeluK, J. L. AND De VRIES, C. G. (2006). Weighted sums of subexponential random variables and asymptotic dependence between returns on reinsurance equities. Insurance Math. Econom. 38, 39-56.

[12] Johg-Dev, K. and Proschan, F. (1983). Negative association of random variables, with applications. Ann. Statist. 11, 286-295.

[13] NG, K. W. AND TANG, Q. (2004). Asymptotic behavior of tail and local probabilities for sums of subexponential random variables. J. Appl. Prob. 41, 108-116.

[14] NG, K. W., TANG, Q. H. AND YANG, H. (2002). Maxima of sums of heavy-tailed random variables. ASTIN Bull. 32, 43-55.

[15] Pakes, A. G. (2004). Convolution equivalence and infinite divisibility. J. Appl. Prob. 41, 407-424.

[16] Peng, L. And Yao, Q. (2004). Nonparametric regression under dependent errors with infinite variance. Ann. Inst. Statist. Math. 56, 73-86.

[17] Petrov, V. V. (1975). A generalization of an inequality of Lévy. Theory Prob. Appl. 20, 141-145.

[18] Rolski, T., Schmidli, H., Schmidt, V. And Teugels, J. (1999). Stochastic Processes for Insurance and Finance. John Wiley, Chichester.

[19] Sgibnev, M. S. (1996). On the distribution of the maxima of partial sums. Statist. Prob. Lett. 28, $235-238$.

[20] TANG, Q. AND YAN, J. (2002). A sharp inequality for the tail probabilities of sums of i.i.d. r.v.'s with dominatedly varying tails. Sci. China Ser. A 45, 1006-1011.

[21] WANG, D. AND TANG, Q. (2004). Maxima of sums and random sums for negatively associated random variables with heavy tails. Statist. Prob. Lett. 68, 287-295.

[22] Willekens, E. (1986). Hogere orde theorie voor subexponentiële verdelingen. Doctoral Thesis, University of Leuven.

[23] Zwart, B., Borst, S. And Mandjes, M. (2004). Exact asymptotics for fluid queues fed by multiple heavy-tailed on-off flows. Ann. Appl. Prob. 14, 903-957. 